MS18-P10

\section{Crystal structure and spectroscopic properties of 1-(pyren-1-yl)but-2-yn-1-one under pressure}

Anna Makal ${ }^{1}$, Joanna Krzeszczakowska ${ }^{1}$

1. Biological and Chemical Research Centre, Chemistry Department, Warsaw, Poland

email: amakal@chem.uw.edu.pl

Controlling properties of materials, such as fluorescence, by subjecting them to macroscopic external physical stimuli (temperature, high-pressure, electric field ...) is a challenge for basic research and an important issue for developing industrial applications. Understanding the mechanisms governing the changes resulting from external stimuli is an essential step in the design of new materials and in optimizing their properties.

Pyrene and its derivatives constitute one of the best studied groups of organic fluorophores[1] characterized by relatively long-lived fluorescence (ns - $\mu$ s excited-state lifetimes), high quantum yields and high environment sensitivity. Multi-substituted pyrene derivatives have already found applications in pressure sensors[2].

One particular property of these fluorophores is their propensity to form excimers. Another is their enhanced solid-state fluorescence. Molecular ordering, resulting from $\pi \ldots \pi$-stacking in the crystal lattice, seems to augment this process. The phenomenon can be considered peculiar, given that clustering of molecules is often raised as the reason for fluorescence quenching in other materials. In light of existing[3] and potential applications of pyrene derivatives in photo-cells and LEDs, a detailed study of the phenomena responsible for efficient fluorescence in solid state seems pertinent. Application of high pressure to the flat, aromatic pyrene derivatives in the crystal lattice should result in forced, closer placement of the aromatic fragments and may be considered as an experimental model for excimer formation, strictly controlled by the applied pressure.

Here we present the results of structural and spectroscopic investigation of a model, simple, monosubstituted pyrene derivative, which in solid state shows 6-fold increase in the quantum efficiency of fluorescence when compared with solution. The crystal structure is based on infinite face-toface $\pi$-stacks of molecules.

Structural analysis at several pressures confirmed minute changes within molecular structure, accompanied by significant modifications of the intermolecular interactions: it was possible to decrease the distance between the flat pyrene moieties by over $0.2 \AA$. These changes were consistent with the changes predicted by means of quantum-mechanical calculations, performed in periodic conditions. The decrease in intermolecular distance can be directly correlated with the decrease of the gap between the valence and conduction bands, marking the compound as an indirect semi-conductor. The changes in the electronic structure find confirmation in the sample's color change under pressure and the spectacular batochromic shift of the fluorescence maximum by $100 \mathrm{~nm}$ between the atmospheric pressure and $3 \mathrm{GPa}$.
References:

[1] J. R.Lakowicz, Principles of fluorescence spectroscopy, Third Edition, Springer, 2008.

[2] M. Sase, S. Yamaguchi, Y. Sagara, I. Yoshikawa, T. Mutai, K. Araki J. Mater. Chem., 2011, 21, 8347.

[3] C.-C. Yu, K.-J. Jiang, J.-H. Huang, F. Zhang, X. Bao, F.-W. Wang, L.-M. Yang, Y. Song Organic Electronics Volume 2013, 14(2), 445 .

Keywords: poly-aromatic hydrocarbons, piezochromism, UVVIS fluorescence 